\title{
Observations of Turbulence Generated by Magnetic Reconnection
}

\author{
J. P. Eastwood, ${ }^{1, *}$ T. D. Phan, ${ }^{1}$ S. D. Bale, ${ }^{1, \dagger}$ and A. Tjulin ${ }^{2}$ \\ ${ }^{1}$ Space Sciences Laboratory, University of California at Berkeley, Berkeley CA 94720, USA \\ ${ }^{2}$ The Blackett Laboratory, Imperial College London, \\ London SWr 2BW, United Kingdom
}

(Dated: December 15, 2008)

\begin{abstract}
Spacecraft observations of turbulence within a magnetic reconnection (guide field $\sim 0$ ) ion diffusion region are presented. In the inertial subrange, electric and magnetic fluctuations both followed a $-5 / 3$ power law; at higher frequencies, the spectral indices were -1 and $-8 / 3$ respectively. The dispersion relation was found to be consistent with fast mode/whistler waves rather than kinetic Alfvén/ion cyclotron waves. Lower hybrid waves, which could be enhanced by whistler mode conversion, were observed but the associated anomalous resistivity was not found to significantly modify the reconnection rate.

PACS numbers: 94.30.cp, 52.35.Vd, 94.30.ct
\end{abstract}

*Electronic address: eastwood@ssl.berkeley.edu

$\dagger$ Also at Department of Physics, UC Berkeley. 
Magnetic reconnection is a fundamental plasma process that enables reconfiguration of magnetic field topology, resulting in the transfer of energy from the magnetic field to the plasma particles themselves [1]. Except for electron-positron plasmas, the diffusion region exhibits a characteristic two scale structure due to differential ion and electron motion [2]. For symmetric inflow conditions, a quadrupole out-of-plane Hall magnetic field pattern is observed, together with a symmetric Hall electric field pointing into the current sheet on both sides [3], and it is thought that this Hall field plays a key role in fast reconnection [4].

However, such simulations typically show that magnetic fluctuations in the ion diffusion region are small, because they cannot describe instabilities that propagate out of the reconnection plane. Recent laboratory experiments appear to show a positive correlation between the reconnection rate and the magnitude of electro-magnetic fluctuations up to the lower hybrid frequency [5], which casts doubt on the role Hall effects play in controlling the rate of fast reconnection. Using small hybrid simulations, it has been concluded that although a turbulent configuration can arise in $3 \mathrm{D}$, this does not significantly enhance the reconnection rate $[6]$.

To establish the role such fluctuations might play relative to Hall physics in controlling reconnection, we present new observations of the fluctuations within a diffusion region in the Earth's magnetotail current sheet made by the four Cluster spacecraft [7] and identified by the presence of Hall electric and magnetic field signatures. The spectral properties of both the electric and magnetic field fluctuations in the diffusion region are presented for the first time, and used to determine the nature of the waves in the dissipation range, a key question being whether energy is deposited in the form of kinetic Alfvén waves [8] or whistler waves $[9]$.

Fig. 1 shows observations of the magnetic field (at 0.045 s resolution) from the FGM experiment [10] together with ion plasma observations (at 4 s resolution) from the CIS experiment [11]. The spacecraft were located at $[-15.6,9.2,3.1]$ Re (Geocentric Solar Magnetospheric coordinates) when they crossed the neutral sheet at 02:25UT. The data are shown in a current sheet coordinate system: the L direction points Earthward and contains the main magnetic field reversal, the $\mathrm{N}$ direction is normal to the plane of the neutral sheet, and $[L, M, N]$ is a right handed triple. Relative to the GSM coordinate system, $\mathrm{L}=$ (0.895, -0.441, 0.068), $\mathrm{M}=(0.445,0.892,-0.072), \mathrm{N}=(-0.029,0.094,0.994)$.

The positive to negative reversal in $B_{L}$ shows that the Cluster spacecraft made a north- 


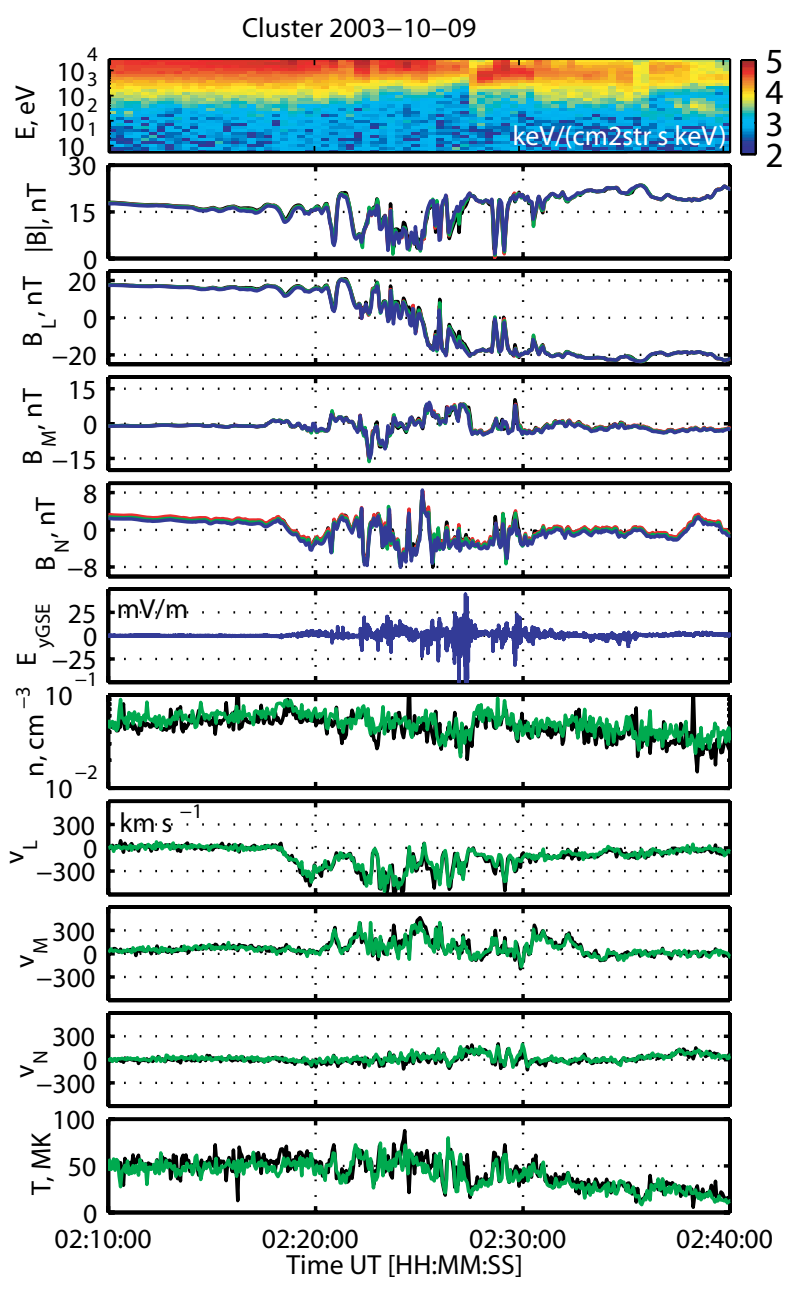

FIG. 1: Cluster observations of the Earth's magnetotail current sheet on 9 October, 2003. Data from the FGM, EFW and CIS experiments are shown. Data from Cluster 1 - 4 are shown in black, red, green and blue respectively. The spectrogram in the top panel is from Cluster 3. Moments of the ion plasma are only available on Cluster 1 and 3 .

south crossing of the magnetotail current sheet (in the direction of their orbital motion; the encounter is shown in Fig. 2). At $\sim 02: 19 \mathrm{UT}$, the spacecraft began to move closer to the neutral sheet, and encountered tailward $\left(-v_{L}\right)$ ion plasma flow, indicating that the X-line was Earthward of Cluster. During this interval, the density and temperature were relatively constant and had values characteristic of the plasma sheet $\left(\mathrm{n}_{i} \sim 0.05 \mathrm{~cm}^{-3}, \mathrm{~T}_{i} \sim 50 \mathrm{MK}\right)$. The spacecraft separation was $\sim 300 \mathrm{~km}$, less than the characteristic ion inertial length $\left(c / \omega_{p i} \sim 1000 \mathrm{~km}\right.$ for $\left.n_{i}=0.05 \mathrm{~cm}^{-3}\right)$. On the scale of Fig. 1 the observations of the four spacecraft are essentially identical. 


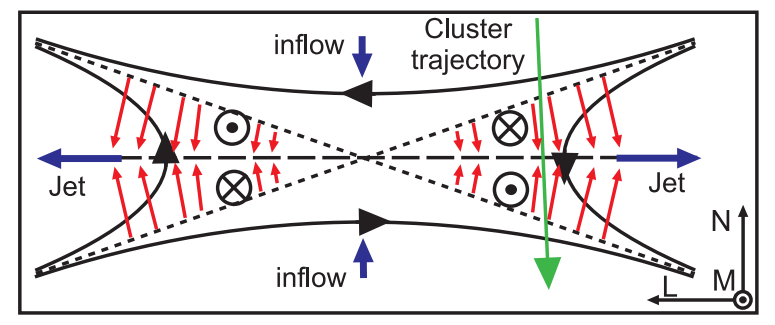

FIG. 2: Cartoon showing the Cluster diffusion region encounter. The magnetic field is shown in black; red arrows show the Hall electric field.

Although on average $B_{M} \sim 0$, indicating little or no guide field, a clear pattern is seen in the perturbations to $B_{M}$ across the current sheet. When $B_{L}>0, B_{M}$ is mainly negative, whereas when $B_{L}<0, B_{M}$ is mainly positive. This is seen more clearly in the top panel of Fig. 3 which shows the correlation between $v_{L}, B_{L}$ and $B_{M}$, measured at 4 s resolution by Cluster 1 and Cluster 3 (the spacecraft for which plasma data is available). In the case of the Hall magnetic field, one expects a quadrupole pattern where $B_{M}$ is negative in the top left and bottom right quadrants, and positive in the other quadrants. The pattern of Hall fields is clearly consistent with that expected, but since the spacecraft encountered only the tailward jet, only half of the quadrupole pattern is observed.

The Hall magnetic field signature should be accompanied by a Hall electric field signature, specifically $E_{N, \text { Hall }}$ should point into the current sheet on both sides where $\mathbf{E}_{\text {Hall }}=(\mathbf{J} \times$ $\mathbf{B} / n e) \sim \mathbf{E}+\mathbf{v} \times \mathbf{B}$. The electric field is measured by the EFW experiment (at a maximum rate of 25 vectors $\mathrm{s}^{-1}$ ) [12]. EFW uses 4 sensors at the end of wire booms to measure the components of the d.c. electric field in the spacecraft spin plane $(\sim$ the Geocentric Solar Ecliptic (GSE) x-y plane). The third component of the electric field (along the spin axis) can be reconstructed using $\mathbf{B}$ (assuming that $\mathbf{E} \cdot \mathbf{B}=0$ i.e. $E_{/ /}=0$ which is expected to be valid everywhere except within the much smaller electron diffusion region), provided $\mathbf{B}$ is not too weak and does not lie in the spin plane. $E_{N, \text { Hall }}$ is negative above the current sheet $\left(B_{L}>0\right)$ and positive below the current sheet $\left(B_{L}<0\right)$, such that $E_{N, \text { Hall }}$ points into the current sheet on both sides. The bottom panel of Fig. 3 shows the correlation between $B_{L}$ and $E_{N, \text { Hall }}$ measured by Cluster 4 (which provides the cleanest measurement of $\mathbf{E})$; the Hall electric field pattern is readily apparent. These observations indicate that the spacecraft were located within the ion diffusion region. We now examine the smaller scale fluctuations in the electric and magnetic field. 


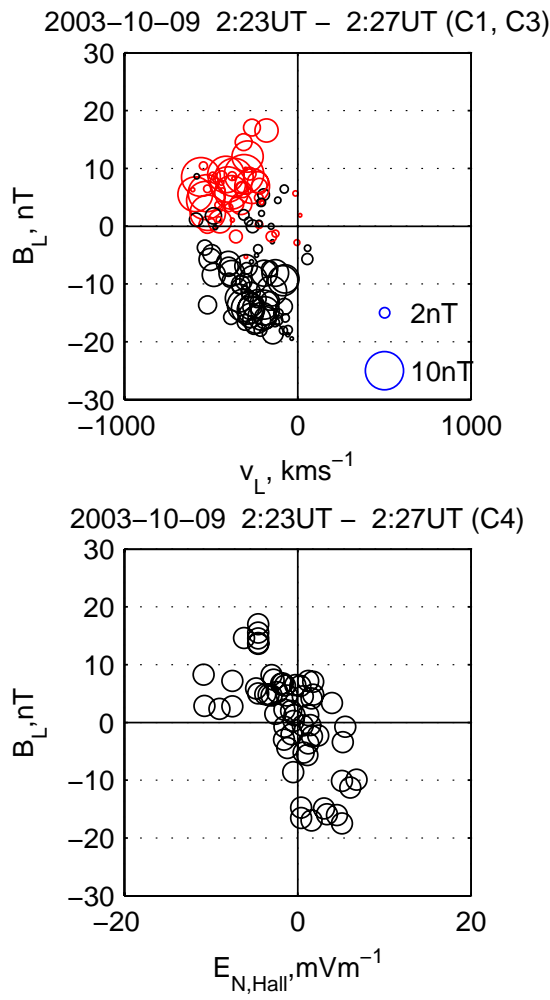

FIG. 3: (top) scatterplot of $v_{L}$ and $B_{L}$ observations made by $\mathrm{C} 1$ and C3 between 02:23UT and 02:27UT. The color of the circles shows the $\operatorname{sign}$ of $B_{M}\left(\right.$ black $=$ positive $B_{M}$, red $=$ negative $\left.B_{M}\right)$. The size of the circle shows the magnitude of $B_{M}$, in comparison to the blue reference circles. (bottom) scatterplot of $E_{N, H a l l}$ and $B_{L}$ as observed by $\mathrm{C} 4$.

Fig. 4 shows the power spectral density (PSD) of the magnetic and electric field measured by Cluster 4 between 02:20 and 02:30UT, calculated using the multi-taper method [13]. The PSD of $E_{y G S E}$ is shown - due to solar illumination effects, this is the best measured component of the electric field [12]. This direction is approximately perpendicular to the outflow. Correspondingly, the PSD of $B_{z G S E}$ is also shown. This is the component of the magnetic field perpendicular to $E_{y G S E}$ and the outflow. Outside the jet, in the reconnection inflow region, no significant wave power is observed in either the magnetic or the electric field.

Between $0.02 \mathrm{~Hz}$ and $0.2 \mathrm{~Hz}$, both the electric and magnetic PSD follow similar power laws. Linear regression [14] shows that the spectral index in this frequency range is $-5 / 3$. Above $0.2 \mathrm{~Hz}$ (which corresponds to the local ion cyclotron frequency), the PSDs diverge; 


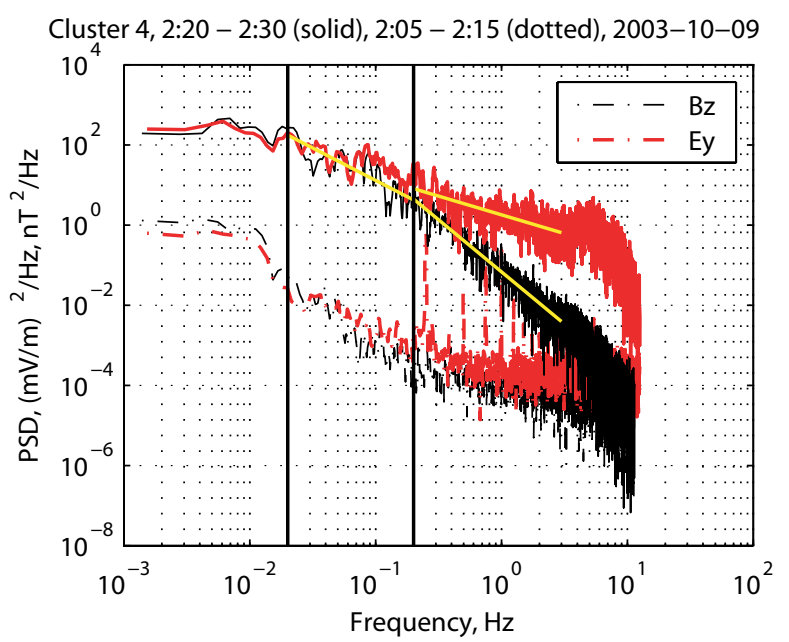

FIG. 4: Power Spectral Density of the $E_{y G S E}$ component of the electric field (red) and the $B_{z G S E}$ component of the magnetic field (black), outside (dashed lines) and inside (solid lines) the jet. The yellow lines show linear fits to the data.

the electric field follows a -1 power law, and the magnetic field follows a $-8 / 3$ power law. We have computed these linear regressions up to a frequency of $3 \mathrm{~Hz}$. Above this frequency, the magnetic field data begins to be affected by 'roll off', where filtering that occurs in the process of measuring the data causes the power to be underestimated at high frequencies. The electric field however displays a distinct enhancement between $3 \mathrm{~Hz}$ and $8 \mathrm{~Hz}$ (not seen in the inflow).

The data can be used to determine the frequency as a function of the wave k-vector, and thus model the dispersion relation of the fluctuations. Using the four spacecraft magnetic field data in combination with the k-filtering technique [15], it is found that the fluctuations are propagating along the outflow direction in the frequency range where the PSDs diverge. This direction is approximately along the reconnecting magnetic field; assuming parallel propagation and using Faraday's law, we find that:

$$
k_{x}=\frac{\omega_{s c}}{v_{s c, x}^{p h}}=2 \pi f_{s c} \times \frac{\delta B_{z}(f)}{\delta E_{y}(f)}
$$

where $v_{s c, x}^{p h}=\omega_{s c} / k_{x}$ is the spacecraft frame phase speed and $\omega_{s c}$ is the spacecraft frame frequency. The phase speed in the jet frame, $v_{j e t, x}^{p h}$, can be estimated by subtracting the average jet speed $\left(v_{j e t} \sim 300 \mathrm{kms}^{-1}\right)$ i.e. $v_{j e t, x}^{p h}=v_{s c, x}^{p h}-v_{j e t}$. Consequently, since $\mathrm{k}$ is frame 


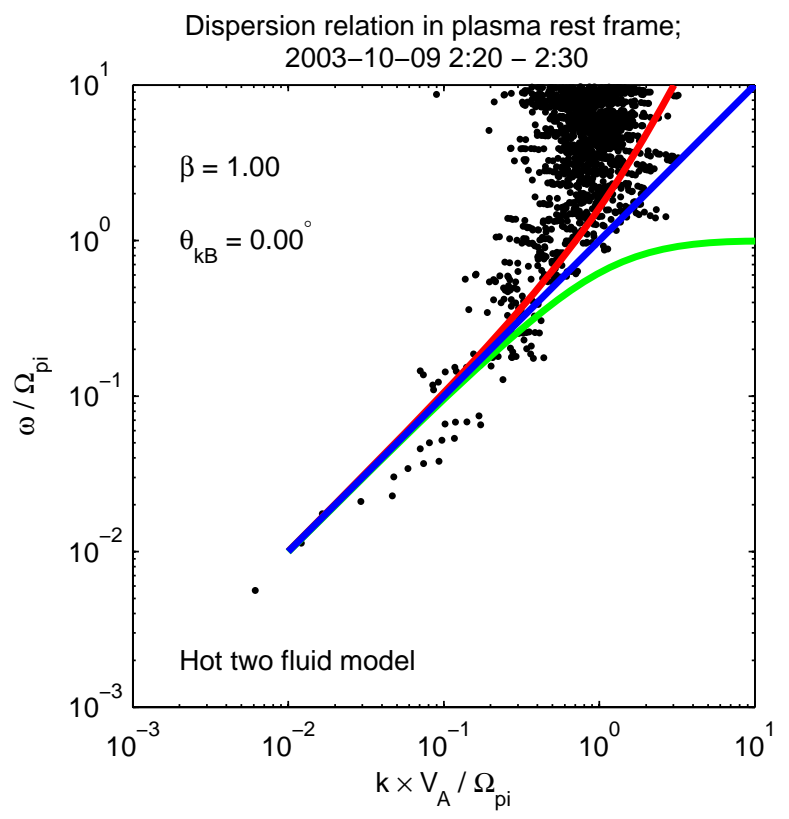

FIG. 5: Dispersion relation derived from the data and model dispersion relations calculated using the hot two fluid approximation.

independent the wave frequency in the jet frame can also be determined as $\omega_{j e t}=v_{j e t, x}^{p h} \times k_{x}$.

Fig. 5 shows how $\omega_{\text {jet }}$ varies as a function of $k_{x}$. Note that both parameters have been normalized. At low frequencies, there is a linear relationship between $\omega$ and $\mathrm{k}$, with the phase speed corresponding to the local Alfvén speed $\left(V_{A, l o c a l}=900 \mathrm{kms}^{-1}\right)$. As $\omega$ approaches $\Omega_{p i}$, the proton gyrofrequency, the dispersion curve bends up, corresponding to an increased phase speed, related to the divergence of the PSDs in Fig. 4 at the frequency where the ions are demagnetizing. For comparison, the hot two fluid plasma dispersion relationship is shown [16]. Free variables in this model are the plasma beta and the angle of propagation. A plasma beta of unity was chosen to compare with the data, and we assume parallel propagation. The red, blue and green lines correspond to the fast (whistler), intermediate (alfvén-ion cyclotron) and slow modes respectively. The data most closely correspond to the whistler mode, although the observed phase speed is higher than the theoretical curve. This may be because the waves are not exactly parallel propagating at some or all of the frequencies under consideration, and the hot two fluid plasma dispersion relations are derived for small amplitude waves in homogenous plasmas.

We now consider the role these fluctuations may play in enhancing resistivity. Applying 
an analysis similar to that of [5] to the magnetic fluctuations, we find that the associated electric field is $\sim 0.3 \mathrm{mVm}^{-1}$, an order of magnitude smaller than the observed reconnection electric field.

The enhancement of only the electric field in the $3-8 \mathrm{~Hz}$ frequency range (Fig. 4), even accounting for the magnetic field roll off, indicates that these fluctuations are electrostatic in nature. This also corresponds to the enhanced fluctuations at around 02:27UT towards the edge of the diffusion region in Fig 1 . The lower hybrid frequency $f_{L H}=\sqrt{\omega_{c i} \omega_{c e}} / 2 \pi=$ $4 \mathrm{~Hz}$ for $\mathrm{B}=6 \mathrm{nT}$, and $6.5 \mathrm{~Hz}$ for $\mathrm{B}=10 \mathrm{nT}$. Thus this enhancement is consistent with lower hybrid wave activity and its expected spatial localization [17-19].

Previous studies $[17,20]$ have concluded that although LH waves can generate anomalous resistivity, they make no significant contribution to the reconnection rate because the LHDI is stabilized in the high plasma beta conditions at the center of the current sheet. Here, although it is possible that whistler waves may be scattering off plasma density variations and converting into lower hybrid fluctuations [21-23], the anomalous resistivity associated with the lower hybrid waves is still found to be small; $\eta \sim 10^{-3} \Omega m$ [24]. Given a cross tail current density of $\sim 10^{-8} \mathrm{Am}^{-2}$, this corresponds to an electric field of $\sim 0.01 \mathrm{mVm}^{-1},(<1 \%$ of the reconnection electric field) meaning that any modification to the overall reconnection rate is negligible.

We have presented observations of the fluctuations within a magnetic reconnection ion diffusion region (with little or no guide field) in the Earth's magnetotail. Turbulent cascades in both the electric and magnetic field fluctuations are inferred from the power law scaling, and energy injected by the reconnection exhaust propagates through the turbulent cascade along the fast mode/whistler branch, providing a new opportunity to study turbulent processes. Lower hybrid waves, perhaps enhanced by the whistler mode conversion, were also observed. The associated anomalous resistivity from both sources was calculated to be small, and so we conclude that whilst dispersive waves may prove to be important in, for example, particle acceleration processes [25], they do not significantly modify the reconnection rate or the Hall physics in magnetotail reconnection. 


\section{Acknowledgments}

This work was supported by NASA grant NNX07AF32G at UC Berkeley. For their comments and discussions about the work we thank T. S. Horbury, C. C. Chaston and F. S. Mozer

[1] V. M. Vasyliunas, Rev. Geophys. 13, 303 (1975).

[2] B. U. O. Sonnerup, Magnetic Field Reconnection (North-Holland, Amsterdam, 1979), pp. $47-108$.

[3] F. S. Mozer, S. D. Bale, and T. D. Phan, Phys. Rev. Lett. 89, 015002 (2002).

[4] J. Birn, J. F. Drake, M. A. Shay, B. N. Rogers, R. E. Denton, M. Hesse, M. Kuznetsova, Z. W. Ma, A. Bhattacharjee, and A. Otto, J. Geophys. Res. 106, 3715 (2001).

[5] H. Ji, S. Terry, M. Yamada, R. Kulsrud, A. Kuritsyn, and Y. Ren, Phys. Rev. Lett. 92, 115001 (2004).

[6] B. N. Rogers, J. F. Drake, and M. A. Shay, Geophys. Res. Lett. 27, 3157 (2000).

[7] C. P. Escoubet, M. Fehringer, and M. L. Goldstein, Ann. Geophys. 19, 1197 (2001).

[8] R. J. Leamon, C. W. Smith, N. F. Ness, and H. K. Wong, J. Geophys. Res. 104, 22331 (1999).

[9] O. Stawicki, S. P. Gary, and H. Li, J. Geophys. Res. 106, 8273 (2001).

[10] A. Balogh, C. M. Carr, M. H. Acuña, M. W. Dunlop, T. J. Beek, P. Brown, K.-H. Fornacon, E. Georgescu, K.-H. Glassmeier, J. Harris, et al., Ann. Geophys. 19, 1207 (2001).

[11] H. Rème, C. Aoustin, J. M. Bosqued, I. Dandouras, B. Lavraud, J. A. Sauvaud, A. Barthe, J. Bouyssou, T. Camus, O. Coeur-Joly, et al., Ann. Geophys. 19, 1303 (2001).

[12] G. Gustafsson, M. André, T. Carozzi, A. I. Eriksson, C. G. Fälthammar, R. Grard, G. Holmgren, J. A. Holtet, N. Ivchenko, T. Karlsson, et al., Ann. Geophys. 19, 1219 (2001).

[13] D. B. Percival and A. T. Walden, Spectral Analysis for Physical Applications (Cambridge University Press, Cambridge, 1993).

[14] J. L. Devore, Probability and Statistics for Engineering and the Sciences (Duxbury, Pacific Grove, CA, 2000).

[15] J.-L. Pinçon and U. Motshmann, Analysis Methods for Multi-Spacecraft Data (International Space Science Institute, Bern, Ch, 1998), chap. 3, pp. 65-78. 
[16] V. Formisano and C. F. Kennel, J. Plasma Phys. 3, 55 (1969).

[17] S. D. Bale, F. S. Mozer, and T. D. Phan, Geophys. Res. Lett. 29(24), 2180, doi:10.1029/2002GL016113 (2002).

[18] P. Petkaki, M. P. Freeman, and A. P. Walsh, Geophys. Res. Lett. 33, L16105, doi:10.1029/2006GL027066 (2006).

[19] T. A. Carter, H. Ji, F. Trintchouk, M. Yamada, and R. M. Kulsrud, Phys. Rev. Lett. 88, 015001 (2002).

[20] R. C. Davidson, N. T. Gladd, C. S. Wu, and J. D. Huba, Phys. Plasmas 20, 301 (1977).

[21] T. F. Bell and H. D. Ngo, J. Geophys. Res. 95, 149 (1990).

[22] J. F. Bamber, W. Gekelman, and J. E. Maggs, Phys. Rev. Lett. 73, 2990 (1994).

[23] T. F. Bell, U. S. Inan, M. Platino, J. S. Pickett, P. A. Kossey, and E. J. Kennedy, Geophys. Res. Lett. 31, L06811, doi:10.1029/2003GL018855 (2004).

[24] F. V. Coroniti, Space Sci. Rev. 42, 399 (1985).

[25] R. Selkowitz and E. G. Blackman, Month. Not. RAS 354, 870 (2004). 